\section{Five-Year Data from the REFLEX Study: A Different Interpretation}

\section{To the Editor:}

I read the 5-year extension data from the REFLEX study ${ }^{1}$ with interest but found the title of the paper - as well as its conclusion - misleading. The authors conclude that rituximab (RTX) re-treatment is associated with maintained or improved efficacy based on clinical responses measured after each of up to 5 courses of RTX. However, there is a fairly pronounced dropout of patients after each course, such that fewer than $20 \%$ of the original cohort ever received a fifth round of the drug. Reasons for dropouts are not detailed, although these data would arguably be the most important information to present and analyze. Instead, the issue of dropout is largely elided in the body of the paper. It appears that the majority of patients withdrew from the study because of "Other reasons," which according to the article's Figure 1 legend, includes insufficient therapeutic response. Knowing the number of patients who discontinued owing to lack of response is of paramount importance (especially as there is no published data on longterm RTX persistence in rheumatoid arthritis) but these data, if known, are not presented.

Taking into account the high dropout rate, the data seem to imply that drug survival for RTX is poor, and that sustained efficacy is the exception rather than the rule. The authors conclude, however, that RTX produces sustained efficacy through data analysis that excludes all patients but an ever-shrinking subcohort still taking the drug. Although the fact that this study is a responder analysis is alluded to in the conclusion of the paper where the shortcomings of the study are listed, the overall thrust of the paper's message is that RTX has prolonged efficacy. However, reaching this conclusion based on measuring clinical efficacy in the minority of patients who have not withdrawn from the study is problematic. It is also somewhat puzzling that in the small group of patients receiving a fifth cycle of RTX - a population highly enriched for clinical response - the American College of Rheumatology 20/50/70 scores are not dramatically better than after cycle one.

In short, although the authors conclude that "RTX repeat treatment leads to clinical efficacy responses that are either maintained or continue to improve over the 5-year period," that appears true, but only in those for whom it works!

ARYEH M. ABELES, MD, Assistant Clinical Professor, Division of Rheumatology, University of Connecticut Health Center, 263 Farmington Avenue, Farmington, CT 06030, USA. Address correspondence to Dr. Abeles; E-mail: aabeles@uchc.edu

\section{REFERENCE}

1. Keystone EC, Cohen SB, Emery P, Kremer JM, Dougados M, Loveless JE, et al. Multiple courses of rituximab produce sustained clinical and radiographic efficacy and safety in patients with rheumatoid arthritis and an inadequate response to 1 or more tumor necrosis factor inhibitors: 5-year data from the REFLEX study. J Rheumatol 2012;39:2238-346.

J Rheumatol 2013;40:5; doi:10.3899/jrheum.121541 